“( 2015 IEEE. Personal use of this material is permitted. Permission from IEEE must be obtained for all other uses, in any current or future media, including reprinting/republishing this material for advertising or promotional purposes, creating new collective works, for resale or redistribution to servers or lists, or reuse of any copyrighted component of this work in other works." 


\title{
Exploiting Unknown Dynamics in Communications Amongst Coexisting Wireless Body Area Networks
}

\author{
Samaneh Movassaghi*, Akbar M. Majidloo ${ }^{\dagger}$, David Smith*, Mehran Abolhasan ${ }^{\S}$, and Abbas Jamalipour $₫$ \\ *National ICT Australia (NICTA) $)^{\ddagger}$, Canberra, ACT, Australia and The Australian National University (ANU), Canberra, Australia \\ ${ }^{\dagger}$ Department of Computer Engineering, Faculty of Technology, Gazi University, Ankara, Turkey

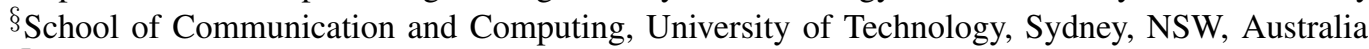 \\ ISchool of Electrical and Information Engineering, The University of Sydney, NSW, Australia \\ Email: (samaneh.movassaghi@nicta.com.au),(a.m.majidloo.gmail.com),(david.smith@nicta.com.au), \\ (mehran.abolhasan@uts.edu.au) and (a.jamalipour@ieee.org)
}

\begin{abstract}
In this paper, we propose a prediction algorithm for dynamic channel allocation amongst coexisting Wireless body area networks (WBANs). Variations in channel assignment due to mobility scenarios within each WBAN as well as the movement of WBANs towards each other is investigated. The proposed scheme is further optimized to allocate the optimum transmission time with synchronous and parallel transmissions such that interference is fully avoided. This reduces the number of interfering nodes and leads to better usage of the scarce limitation of resources in these networks, larger network lifetime, higher energy savings and higher throughput. In fact, the aim of this protocol is to mitigate interference along with maintaining minimum power consumption in order to maximize network lifetime and increase the spatial reuse and throughput of each WBAN. Simulation results show that our approach achieves a much higher spatial reuse using the smart spectrum allocation scheme for interference mitigation in collocated WBANs. We conduct extensive simulations for coexistence prediction in different mobility scenarios using the NS-2 simulator. Consequently, we demonstrate the efficiency of the proposed protocol in providing interference-free channel assignments and higher energy savings.

Index Terms-Wireless Body Area Networks, Spectral Efficiency, IEEE 802.15.4, Interference Mitigation, WBANs
\end{abstract}

\section{INTRODUCTION}

WBANs are cyber-physical systems that are designed to provide a vast era of applications from real-time health care to personal entertainment services. They cannot be considered as static networks therefore they are subject to change and must be able to cope with the variations in their network. These variations can be in terms of topological changes, variations in traffic patterns, changes in overall load on the network. Additionally, the environment in WBANs is non-deterministic which implies taking the same action on the same state for two different occasions may lead to different states. Therefore adaptive spectrum allocation techniques must be deployed in these networks to take these unknown dynamics into account. However, adaptive approaches require frequent exchange of information and lead to a linear cost in updating information. In addition, the broadcast nature of the wireless spectrum and the

\footnotetext{
${ }^{\ddagger}$ NICTA is funded by the Australian Government as represented by the Department of Broadband, Communications and the Digital Economy and the Australian Research Council through the ICT Centre of Excellence program.
}

limited radio bandwidth leads to interference between devices involved in communication between coexisting WBANs. More specifically, the same PHY technology cannot be used for simultaneous transmissions over two or more conflicting wireless links. In addition, power is a scarce resource in WBANs as sensors are battery-driven. Moreover, future WBANs are prone to excessive interference in densely populated areas which can significantly degrade network performance and quickly depletes the energy of WBAN nodes. This issue is highlighted even in medical applications that deal with mission-critical information where unreliable data collection endangers the life of millions of people. Therefore, interference mitigation is of utmost importance amongst coexisting WBANs to minimize system power consumption and increase the reliability of the system.

The challenges of interference mitigation in WBANs are as follows: 1) Interference on a single node depends on the independent decisions made by multiple coordinators; 2 ) The system architecture is decentralized as the no central entity can provide global control in terms of interference on the nodes of different WBANs; 3) Due to the increase in the number of nodes in a WBAN a solution based on a centralized agent will not be scalable; 4) Individual decisions of each WBAN coordinator has to be self-adaptive based on the decisions of the other coordinators and the surrounding environment. 5) Coexisting WBANs use several transmission technologies that share the same unlicensed band (ISM band) which leads to a dramatic increase on the level of interference of coexisting WBANs as well as their network performance. However, most interference mitigation proposals for WBANs [1-8] have only considered to optimize only one of these goals; whilst all requirements must be addressed as a whole in the design of an efficient interference mitigation scheme for WBANs. Also, they do not consider the social nature of WBANS [9]. The rest of the paper is organized as follows. The system model is described in Section II. The proposed prediction scheme is proposed in section III. Simulation and analytical results are provided in Section IV. Finally, conclusions are drawn in Section V. 


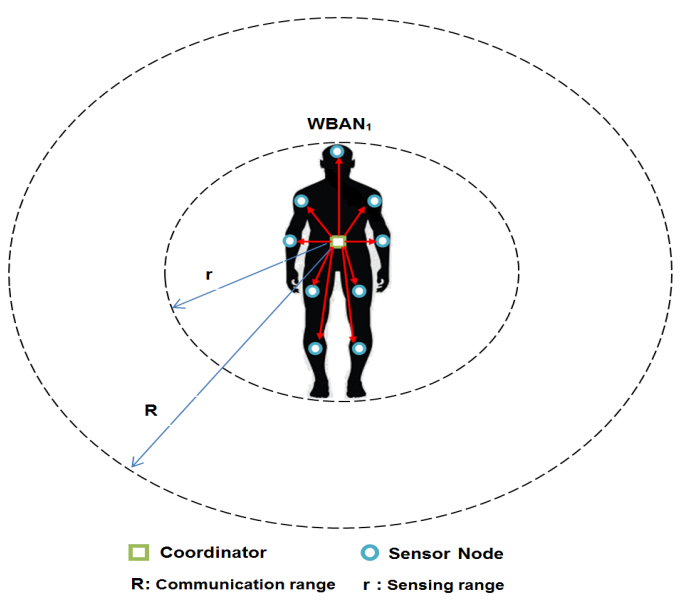

Fig. 1. System Diagram of WBANs

\section{SySTEM MODEL}

We consider a set of coexisting WBANs denoted by $\left\{w_{k} \mid k=1, \ldots, M\right\}$, where $M$ is the number of WBANs and $w_{k}$ refers to the $k$ th WBAN. Each WBAN is composed of a homogenous set of $N$ sensors denoted by $\left\{s_{k}^{i} \mid i=1, \ldots, N\right\}$ of equal importance and one coordinator node as the receiver placed in the center. For simplicity, we model each WBAN by two concentric circles, one of radius $r$ known to be its sensing range, and one of radius $R$, known as its communication range, shown in Fig. $1(R=2 r)$. Sensor nodes are uniformly located at random within the circle.

All WBANs have use similar superframe structure and interWBAN superframe synchronization is achieved before transmission. Thus, collisions never occur between one WBAN's control frame and another WBAN's data frame. Whilst interference may occur between coexisting WBAN's data frames in the Contention Free Periods (CFP) [10]. We assume that all sensor nodes have similar characteristics, a unique identification number (ID) and are initially provisioned with an equal amount of energy. The coordinator broadcasts a message in the $R$ radius coverage area containing the ID of its sensor nodes, their residual energy, location in the buffer and their allocated time slot.

Nodes within each WBAN follow TDMA for channel assignment which implies each sensor node is allocated an equal amount of time for its transmissions. Thus, in order to avoid interference, nodes that exist in the interference set of one another need to be allocated orthogonal channels that do not coincide with one another's transmission. Hence, the placement of nodes in the buffer needs to be taken into account to avoid any mistakes with channel allocation.

After the first round of data transmission, the change in positioning of sensor nodes throughout the network can be realized, through which sensor nodes that are static can be told apart from those that are dynamic. More specifically, the nodes which have moved closer to the $r$ region can be discovered. For instance, if a sensor node is within $190 \mathrm{~cm}$ of the $R$ region, there is a huge difference to when it is withing $110 \mathrm{~cm}$ of the

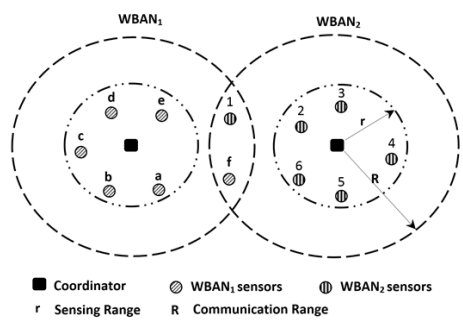

Fig. 2. An example of distribution of nodes amongst two WBANs and their Interference Region

$R$ region, which implies it is only $10 \mathrm{~cm}$ far from the $r$ radius region. This way, sensor nodes which are more likely to join the inter-WBAN interference set can be known beforehand and planning can be done in advance before any interference is acknowledged.

\section{Proposed Prediction Algorithm Amongst COEXISTING WBANs}

\section{A. Smart Channel Assignment (SCA) Technique}

Coexisting WBANs initially use the authors Smart Spectrum Allocation technique proposed in [11] briefly described as follows:

\section{- Step 1. Orthogonal Transmission}

Coordinators of coexisting WBANs negotiate to assign orthogonal channels for each WBAN. So, the shared channel is evenly divided into $N_{c} N_{s}$ for each sensor node where the $\ell^{\text {th }}$ sensor of $W B A N_{i}$ transmits at time slot $T_{i, \ell}$. Meanwhile, collocated coordinators compute the interference level of the transmission of that sensor on its own sensors from the received signal power. Let $\gamma_{i, j, \ell}$ denote the received power from the $\ell^{t h}$ sensor of $W B A N_{j}$ at $W B A N_{i}$. After orthogonal transmission of all sensors in the first round, each coordinator creates a table consisting of the received power from each sensor of all WBANs.

\section{- Step 2. Formation of the Interference Set}

In the second round of transmission, each coordinator finds the minimum received power from its sensors in $W B A N_{i}$ at its coordinator referred to as $\gamma_{m i n, i}$. If the received power of a sensor from other WBANs is larger that $\gamma_{\min , i}-\gamma_{T h}$, that sensor is added to the Inter-Interference list shown as follows:

$$
\mathcal{I}_{i}=\left\{(j, \ell) \mid \gamma_{i, j, \ell}>\gamma_{\min , i}-\gamma_{T h}\right\}
$$

- Step 3. Exchanging Information

Each coordinator broadcasts its interference list at this stage. Therefore, each coordinator can determine which of its sensors interfere on the transmission of other WBANs and which of its own sensors impose interference on other WBANs. Then the coordinator of each WBAN, $W B A N_{i}$, creates an interference set as follows:

$$
\mathcal{S}_{i}=I_{i} \cup\left\{(i, \ell) \mid(i, \ell) \in \mathcal{I}_{j}, j \neq i\right\}
$$




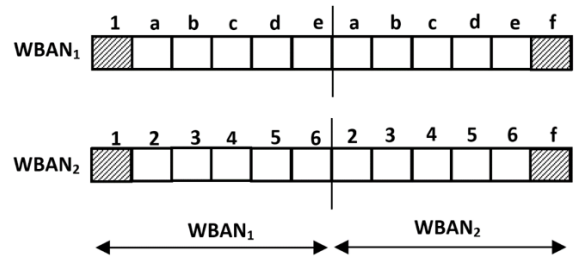

Fig. 3. Channel Assignment using the SCA technique for nodes in Fig. 2.

\section{- Step 4.Spectrum Allocation}

Then, each WBAN assigns channels to its sensors as shown in Fig. 3 by allocating orthogonal channels to nodes in the interference set and dividing the rest of the channels amongst other nodes within each WBAN.

\section{B. Proposed Prediction Algorithm for Unknown Dynamism}

During the first round of orthogonal transmissions each WBAN also informs coexisting WBANs of its sensors unique identification address, localization information and residual energy. Each WBAN stores the minimum received power of its sensors at the previous time slot and the current time slot. In the case that, the received power at the time instant $t$ is similar to time instant $t-1$ and still in the range of above threshold received power or below that, there will not be any changes in channel assignment. Whilst, in the case that the distance of a sensor minus the radius of the sensing range is less than zero, the sensor node has moved into the $\mathrm{R}$ region. If the received power of the node at time instant $t$ has increased from its value at time instant $t-1$ that node is moving close to the $r$ region. Therefore, it is added to the interference set and smart channel assignment is updated accordingly. In the case that the received power of a node at time instant $t$ has decreased from its value at time instant $t-1$ that node is moving out to the $R$ region, is removed from the interference set and smart channel assignment is updated accordingly. The pseudo code for the proposed prediction algorithm for capturing unknown dynamics amongst coexisting networks in shown in Algorithm. 1.

In the case that we have $x$ nodes in the network, we will need $x$ timeslots in each period to allocate to the $x$ nodes. The allocations can be made through $x$ ! functions shown with $f$ and $g$ for two coexisting WBANs. Let us consider that in a period $T$, the function $f$ has been used to map the $x$ available timeslots to the $x$ number of nodes as follows:

$$
\left(t_{1}, t_{2}, \ldots, t_{x}\right) \stackrel{f}{\rightarrow}\left(N_{1}, N_{2}, \ldots, N_{x}\right)
$$

The function $g$ has been used in the same period $T$ to map its nodes to the allocated timeslots as follows:

$$
\left(t_{1}, t_{2}, \ldots, t_{x}\right) \stackrel{g}{\rightarrow}\left(N_{1}, N_{2}, \ldots, N_{x}\right)
$$

In the case that the two functions do not overlap we do not have any changes within the network, however, if there is an overlap between two specific nodes for instance $N_{2}$ and $N_{5}$, which means the value of the function $f$ is equal in $\left\{t_{2}, t_{5}\right\}$, this implies we have interference: $f \mid t_{1}, t_{2}=g$.

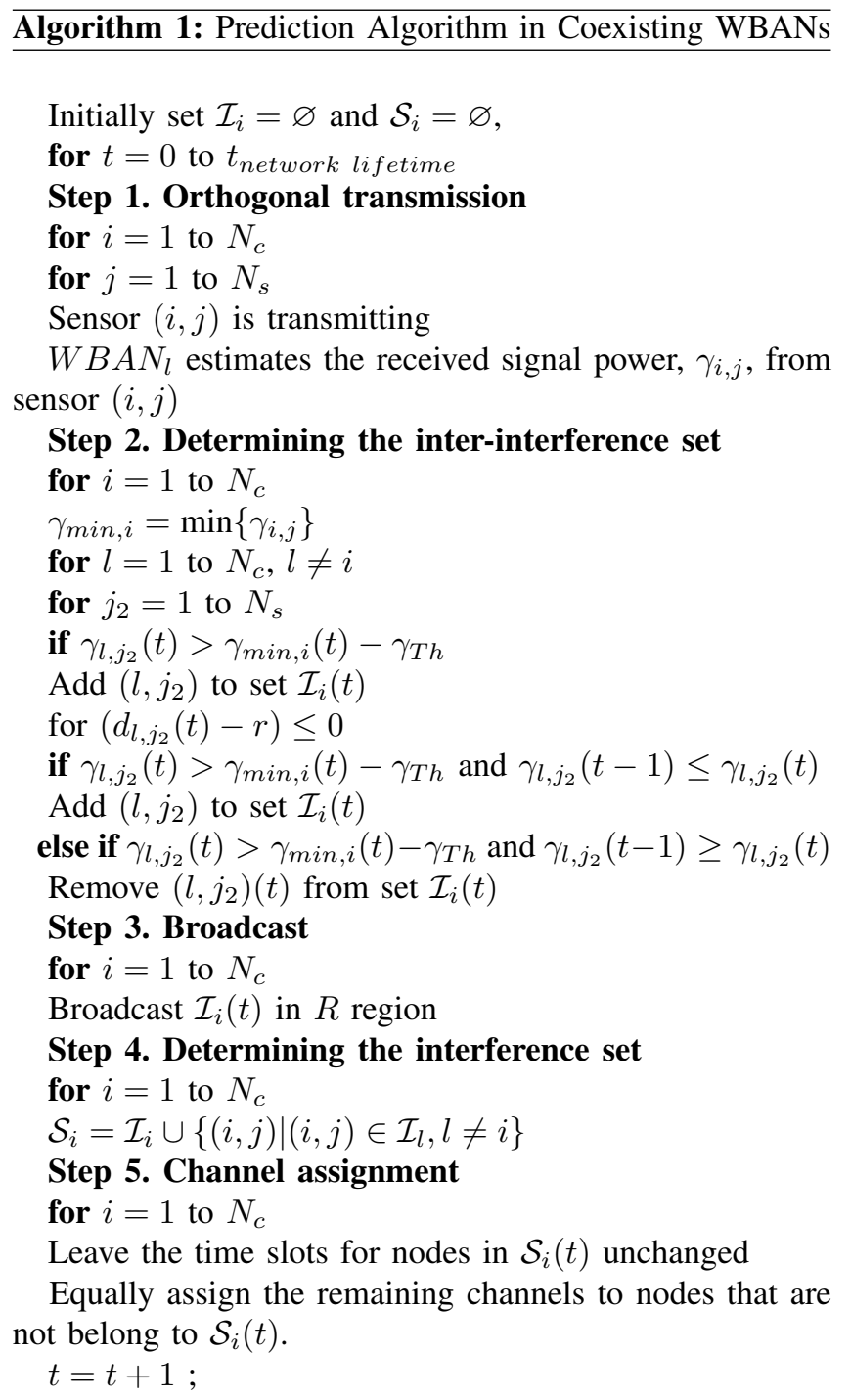

\section{Mobility Scenarios Amongst Coexisting WBANS}

As sensor nodes in WBANs have high mobility, their link connectivity and Packet Delivery Ratio (PDR) varies between different postures, the proposed channel allocation scheme should support variations in link quality and network topology. Thus, we have chosen the mobility model proposed in relative to mobility in different postures and individual mobility within a specific posture. Therefore, we evaluate the smart spectrum allocation for the following coexistence environments:

\section{A. Scenario 1}

Two WBANs $\left(W B A N_{1}\right.$ and $\left.W B A N_{2}\right)$ are on a straight line and walking back to back to each other. If the speed of $W B A N_{1}$ which is ahead, is more than or equal to the 
$W B A N_{2}$ behind it there will be no interference between them.

$$
\begin{array}{r}
\forall t: I f \quad V_{W B A N_{1}} \geq V_{W B A N_{2}} \\
\Rightarrow r_{A} \cap r_{B}=\emptyset
\end{array}
$$

However, in the case where the speed of the WBAN at the back is higher than the one in front, the scenario will be very similar to the second subscenario with the difference of having a lower time in the intersection region which is a function of the speed of the two WBANs $\left(r_{A}=r_{B}=r\right)$. If $V_{W B A N_{1}} \leq V_{W B A N_{2}}$ then:

$$
\begin{array}{r}
d-2 r=\left(V_{W B A N_{2}}-V_{W B A N_{1}}\right) \times t \\
\Rightarrow t=\frac{d-2 \times r}{\left(V_{W B A N_{2}}-V_{W B A N_{1}}\right)}
\end{array}
$$

The $t$ found from the equation above is the exact time the interference initiates.

$$
\begin{gathered}
4 r=\left(V_{W B A N_{2}}-V_{W B A N_{1}}\right) \times t^{\prime} \\
\Rightarrow t^{\prime}=\frac{4 \times r}{\left(V_{W B A N_{2}}-V_{W B A N_{1}}\right)}
\end{gathered}
$$

where $t^{\prime}$ calculated in the equation above is the actual time duration in which there is a probability for interference which obviously starts after $t$.

\section{B. Scenario 2}

Two WBANs are walking on a straight line with similar or different speeds towards each other. The calculations for this scenario are just like the previous state with the only difference that the speed of the moving WBAN would be the summation of the speed of the two WBANs. Thus, the time of at which interference between two coexisting WBANs initiates is a function of the speed of the collocated WBANs and can be calculated as follows:

$$
\begin{array}{r}
d-2 r=\left(V_{W B A N_{2}}-\left(-V_{W B A N_{1}}\right)\right) \times t \\
\Rightarrow t=\frac{d-2 \times r}{\left(V_{W B A N_{2}}+V_{W B A N_{1}}\right)}
\end{array}
$$

The duration at which there is a likelihood for the coexisting WBANs to interfere with each other, referred to as $t^{\prime}$ can be calculated as follows:

$$
\begin{gathered}
4 r=\left(V_{W B A N_{2}}+V_{W B A N_{1}}\right) \times t^{\prime} \\
\Rightarrow t^{\prime}=\frac{4 \times r}{\left(V_{W B A N_{2}}+V_{W B A N_{1}}\right)}
\end{gathered}
$$

\section{Scenario 3}

In this scenario, we consider two coexisting WBANs, where $W B A N_{1}$ is static whilst $W B A N_{2}$ has dynamics and approaches $W B A N_{1}$ with a speed of $V$. The time at which it reaches the $2 R$ region and the duration at which it will stay in that region can be calculated as follows:

$$
4 R=V \times t \Rightarrow t=\frac{4 R}{V}
$$

It is obvious that the higher the speed the lower the time of interference of the two WBANs. More importantly, we only

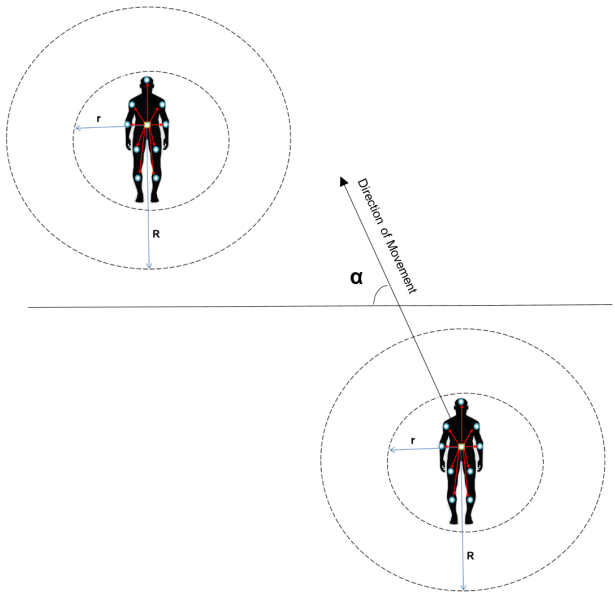

Fig. 4. Movement of two WBANs with respect to each other

need to use the proposed SCA algorithm in this time. This implies that coexisting WBANs can transmit in parallel which leads to significantly higher throughput.

Lemma: If the calculated time of interference is less than a period, no no interference will take place. However, in the case where the ID of the node in the intersection is similar to the ID of the transmitting node, interference cannot be avoided.

\section{Scenario 4}

In this scenario, we consider two coexisting WBANs, where $W B A N_{1}$ is static whilst $W B A N_{2}$ has dynamics and approaches $W B A N_{1}$ with a speed of $V$ and an angle of $\alpha(\alpha$ is the angle between the direction of movement of $W B A N_{2}$ and the horizontal axis of movement shown in Fig.4).

The important aspect of this scenario is to discover if the two WBANs will interfere on one another's transmission having this angle between the direction of movement of the two WBANs. If there is a chance they would interfere on each other's transmission, then we can avoid their interference with the SCA technique.

We consider $(a, b)$ to be the coordinates of the center of $W B A N_{1}$. Since $\tan (\pi-\alpha)=-\tan (\alpha)$, we have the following:

$$
\begin{gathered}
y=-\tan (\alpha) \times x+c \\
m=\frac{-1}{-\tan (\alpha)}=\cot (\alpha) \\
y-a=\cot (\alpha)(x-b)=\cot (\alpha) x-\cot (\alpha) b+a
\end{gathered}
$$

By substituting EQ.(11) into EQ.(13) we have the following:

$$
-\tan (\alpha) \times x+c=\cot (\alpha) x-\cot (\alpha) b+a
$$

Since the values of $a, b$ and $c$ are known in advance, we can find the value of $x$ and $y$. We can also calculate $d$ as follows:

$$
d=\sqrt{(x-a)^{2}+(y-b)^{2}}
$$


If $d>2 R$ we do not have intersection of regions between the coexisting WBANs. If $d<2 R$, the coexisting WBAN intersect in their regions where the equation $2 R-d$ shows the extent of their intersection. The higher this value, the more the interference. If $d=0$ their intersection ratio will be $100 \%$. The value of $d$ also shows how far or how close we are to the $r$ region $(d<2 r$ and $d>2 r)$. Based on the aforementioned calculations, the WBANs will realize if they would need to make any changes to their assigned allocations.

The amount of time the coexisting WBANs remain in each others coexistence can be realized from the intersection of the two coexisting circles at two points which can be found from solving the two equations of the two intersecting circles with radius $\mathrm{R}$ and with the centers of (x,y) and (a,b). Based on the distance between the two nodes, $d$, their intersection time can be calculated as follows:

$$
d=V \times t \Rightarrow t=\frac{d}{V}
$$

It is important to note that $\alpha$ never equals zero in this equation since $m_{1} \times m_{2}=-1$ where $m_{1}=m$ and $\tan (\alpha)=m_{2}$. If $\alpha=0$ :

$$
m=\frac{-1}{-\tan (0)}=\cot (0)=\infty
$$

which provides us with an invalid result. Thus, we have described the case for $\alpha=0$ separately in Scenario 2 .

\section{Simulation Results}

In order to evaluate the performance of the proposed prediction algorithm, we have performed simulation of the proposed scheme in NS-2 simulator (version 2.34) [12] with a one hop star topology within each WBAN for four different coexistence scenarios described in Section IV. In this section, we compare the performance of the proposed scheme in the aforementioned mobility scenarios with the 802.15.4 protocol which considers TDMA for inter-WBAN communication in terms of delay, throughput, PDR and energy consumption. The parameters used for these simulations are provided in Table. I.

TABLE I

PARAMETERS IN SiMUlation

\begin{tabular}{cc}
\hline \hline Simulator & NS-2 \\
Simulation duration (Sec) & 120 \\
Simulation area & $20 \mathrm{~m} \mathrm{X} 20 \mathrm{~m}$ \\
Number of Sensor Nodes per WBAN & 9 \\
Number of Coordinators per WBAN & 1 \\
Number of WBANs & 2 \\
MAC Layer Protocol & IEEE 802.15 .4 \\
Queue Size & 50 \\
Packet rate & 4 packets/sec \\
Packet Size & $512 \mathrm{Bytes}$ \\
Sensing Range & $100 \mathrm{~cm}$ \\
Initial Energy & $50 \mathrm{~J}$ \\
\hline \hline
\end{tabular}

Fig .5 shows the average sensor node consumable energy versus the simulation time for the four different scenarios compared to IEEE 802.15.4. As can be clearly seen in this figure, the proposed scheme provides more energy savings

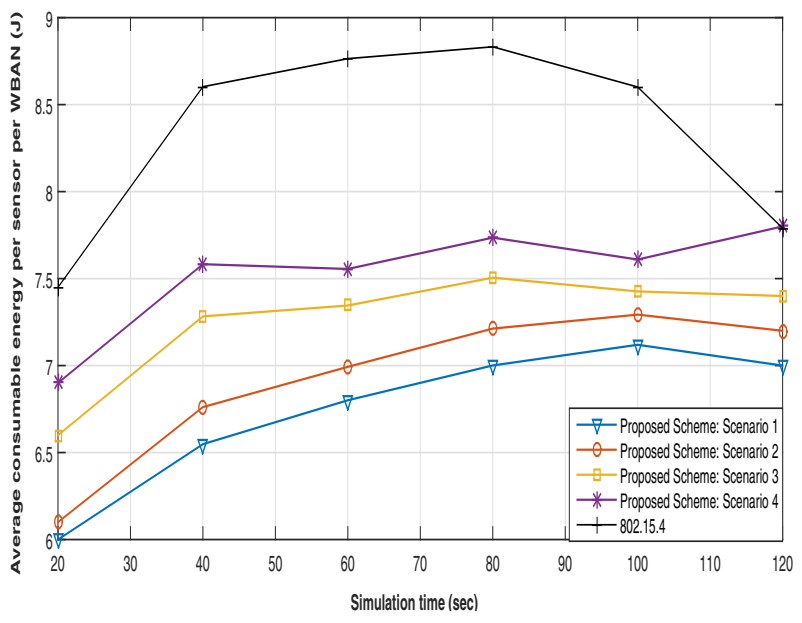

Fig. 5. Average Consumable Energy versus Simulation Time

which is because of reduced interference that leads to energy wastage. After a number of transmissions, the proposed prediction algorithm can reach up to 50\% energy savings compared to IEEE 802.15.4. This feature is quite beneficial to such networks with stringent battery requirements as battery replacements can be quite uncomfortable.

Fig. 6 depicts the average delay of each sensor per WBAN for transmission versus the simulation time for two coexisting WBANs, when $N_{s}=9$. It is important to note that in conventional approaches, nodes in the network orthogonally transmit, which means that the overall time frame is divided into $2 \times N_{s}$ equal length time slots, and each sensor transmits in only one of these time slots and remains silent in remaining $2 \times N_{s}-1$ time slots. Such an approach leads to a huge delay, which is very inefficient especially in emergency applications of WBANs where mission-critical data needs to be sent. As can be seen, initially all four scenarios of the prediction algorithm and the IEEE 802.15.4 protocol have similar delay since they will all be using orthogonal transmission in their first round, whilst after that, the delay for the IEEE 802.15.4 protocol is $45 \%$ higher than when the proposed prediction algorithm is used for inter-WBAN communication.

the proposed approach maintains a reasonably lower delay in all four scenarios.

Finally, Fig.7 and Fig.8 demonstrate that our proposed scheme achieves an optimal packet delivery ratio and throughput compared to the existing standard. This is shown to increase by the simulation time at an order of 8 in the PDR and an order of 5 in terms of throughput which is quite massive in the case of WBANs with high data rate requirements and low energy supplies.

\section{CONCLUSION}

We have proposed a decentralized prediction algorithm for inter-WBAN communications using the smart channel assignment technique. Specifically, the proposed scheme captures the unknown dynamics and provides feedback to the coordinator 


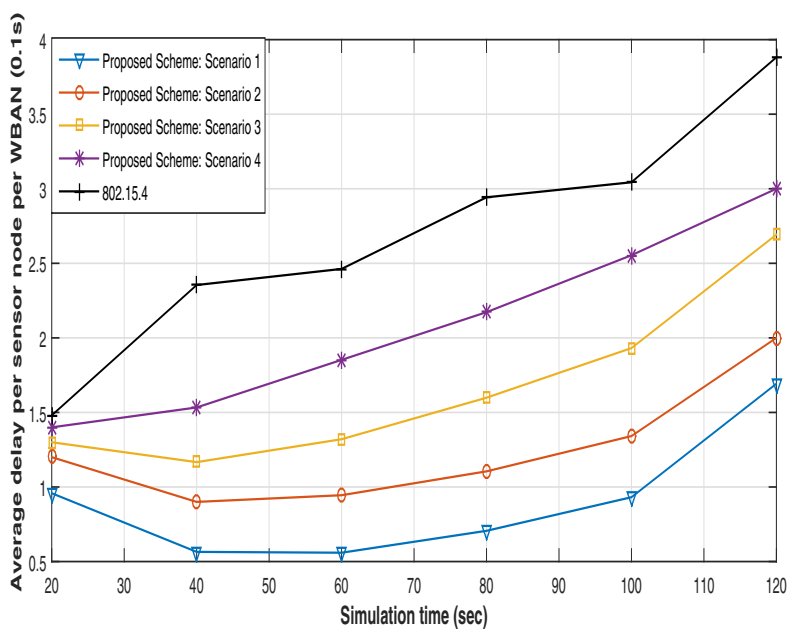

Fig. 6. Average Delay versus Simulation Time

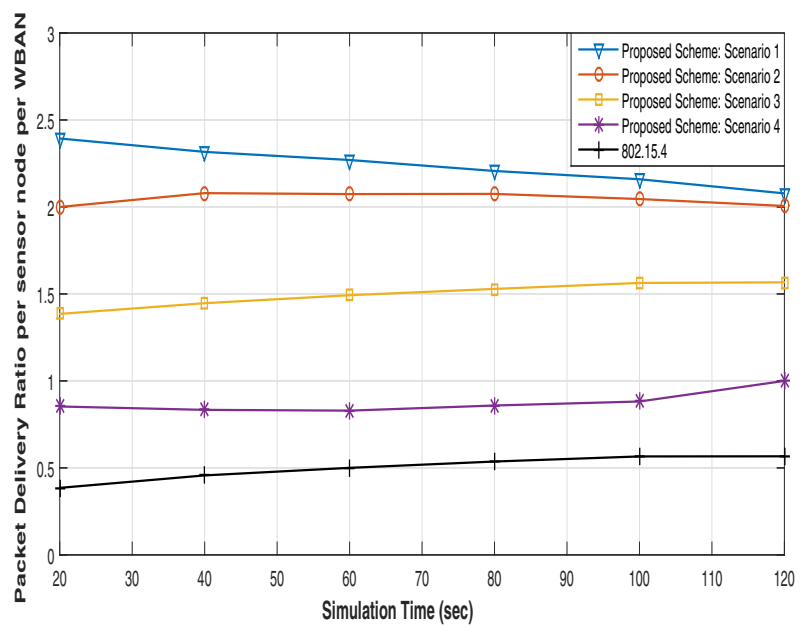

Fig. 7. Average Packet Delivery Ratio versus Simulation Time

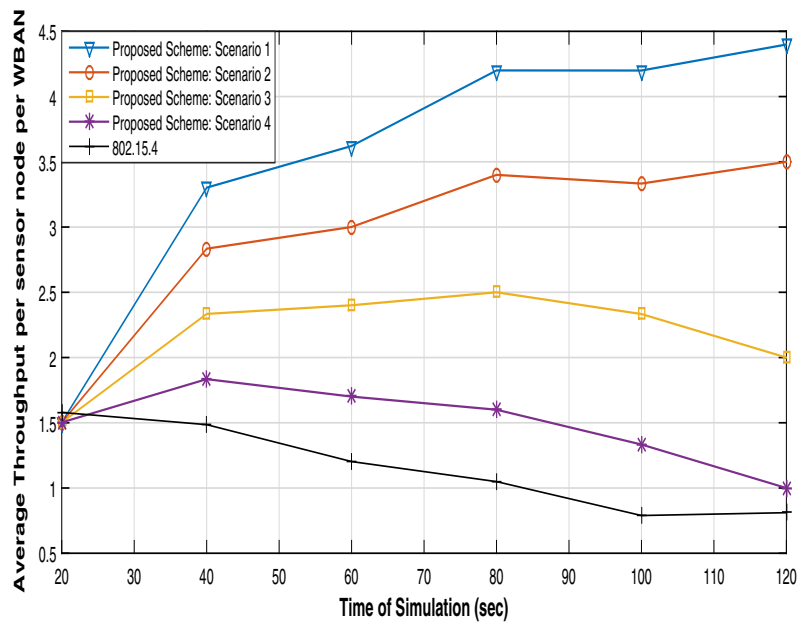

Fig. 8. Average Throughput versus Simulation Time of each WBAN and quickly updates its channel assignment based on the recent changes in the network. Theoretical analysis towards computation of the time instant at which coexisting networks initiate imposing interference on one another and the duration that it lasts. Simulation results demonstrate that the proposed approach achieves more efficient usage of the scarce limitation of resources in WBANs which leads to major energy savings leading to a higher network lifetime. Additionally, simultaneous transmissions in coexisting WBANs allows for higher throughput and packet delivery ratio. We have shown that the proposed scheme can optimal update channel assignment to keep the interference level below the desired threshold. Simulation results have shown the efficiency of the proposed approach through four different mobility scenarios compared to the IEEE 802.15.4 approach. To conclude, the major advantages of the proposed approach are extended network lifetime, massive energy savings and significantly higher throughput through four different mobility scenarios.

\section{REFERENCES}

[1] A. Zhang, D. B. Smith, D. Miniutti, L. W. Hanlen, D. Rodda, and B. Gilbert, "Performance of piconet co-existence schemes in wireless body area networks," in IEEE Wireless Communications and Networking Conference (WCNC), 2010, pp. 1-6.

[2] J. Dong and D. Smith, "Cooperative body-area-communications: Enhancing coexistence without coordination between networks," in IEEE 23rd International Symposium on Personal Indoor and Mobile Radio Communications (PIMRC), 2012, pp. 2269-2274.

[3] W.-B. Yang and K. Sayrafian-Pour, "Interference mitigation for body area networks," in 22nd IEEE International Symposium on Personal Indoor and Mobile Radio Communications (PIMRC), 2011, pp. 2193 2197.

[4] D. Smith, L. Hanlen, D. Miniutti, J. Zhang, D. Rodda, and B. Gilbert, "Statistical characterization of the dynamic narrowband body area channel," in 1st IEEE International Symposium on Applied Sciences on Biomedical and Communication Technologies (ISABEL'08), 2008, pp. $1-5$.

[5] B. de Silva, A. Natarajan, and M. Motani, "Inter-user interference in body sensor networks: Preliminary investigation and an infrastructurebased solution," in 6th IEEE International Workshop on Wearable and Implantable Body Sensor Networks (BSN), 2009, pp. 35-40.

[6] P. R. Grassi, V. Rana, I. Beretta, and D. Sciuto, " $B^{2}$ IRS: A technique to reduce ban-ban interferences in wireless sensor networks," in 9th IEEE International Conference on Wearable and Implantable Body Sensor Networks (BSN), 2012, pp. 46-51.

[7] S. Cheng and C. Huang, "Coloring-based inter-wban scheduling for mobile wireless body area network," IEEE Transactions on Parallel and Distributed Systems, 2013.

[8] X. Wang and L. Cai, "Interference analysis of co-existing wireless body area networks," in IEEE Global Telecommunications Conference (GLOBECOM 2011), 2011.

[9] Z. Zhang, H. Wang, C. Wang, and H. Fang, "Interference mitigation for cyber-physical wireless body area network system using social networks," IEEE Transactions on Emerging Topics in Computing, vol. 1, no. 1 , pp. 121-132, 2013.

[10] L. Wang, C. Goursaud, N. Nikaein, L. Cottatellucci, and J. Gorce, "Cooperative scheduling for coexisting body area networks," IEEE Transactions on Wireless Communications, vol. 12, no. 1, pp. 123-133, 2013.

[11] S. Movassaghi, M. Abolhasan, and D. Smith, "Smart spectrum allocation for interference mitigation in wireless body area networks," in IEEE International Conference on Communications (ICC), 2014.

[12] UCB/LBNL/VINT, “Network simulator-ns," April. 2015. [Online]. Available: http://wwwmash.cs.berkeley.edu/ns 